\title{
AM2020-5050: AN ELLIPTICAL GALAXY WITH AN OUTER RING
}

\author{
Bradley Whitmore, \\ Doug McElroy \\ Space Telescope Science Inst. \\ 3700 San Martin Drive \\ Baltimore, MD 21218
}

\author{
François Schweizer \\ Dept. of Terrestrial Magnetism \\ Carnegie Inst. of Washington \\ 5241 Broad Branch Rd. NW \\ Washington, DC 20015
}

\begin{abstract}
Photometric and spectroscopic observations show that the inner component of AM2020-5050 is an elliptical galaxy, unlike other polar-ring galaxies which have an S0 disk at the center. A comparison of the central velocity dispersion with the rotational velocity in the ring suggests the presence of a nearly spherical gravitational potential. The inner component has a rapidly rotating core with rotational velocities at $3^{\prime \prime}$ substantially higher than at $8^{\prime \prime}$. Although the optical ring is quite narrow, $\mathrm{H}_{\alpha}$ emission is observed all the way through the center of the galaxy, indicating tine presence of an extended gaseous disk.
\end{abstract}

\section{OBSERVATIONS AND RESULTS}

Observations have shown that the central components of polar-ring galaxies tend to be highly inclined S0 disks, with a large rotational velocity and small velocity dispersion. Photographs of the polar-ring galaxy AM2020-5050 led us to believe that the central component in this case might be an elliptical galaxy. We obtained photometric and spectroscopic observations using the 4 meter telescope at CTIO to check this hypothesis.

Photometry in the $\mathrm{B}$ and $\mathrm{V}$ bands with a CCD detector at prime focus shows that the luminosity profile follows an $\mathrm{R}^{\frac{1}{4}}$ law characteristic of an elliptical galaxy (see Figure 1), rather than the exponential law seen in most disk galaxies. The ring is bluer than the central component with $B-V=0.6$ compared to $B-V=0.9$.

Long-slit spectra obtained in the blue $(4100-5600 \AA)$ show that the central component rotates quite slowly at $8^{\prime \prime}$, unlike S0 disks which rotate rapidly. The ratio of the rotational velocity to the central stellar velocity dispersion is $\mathrm{V} / \sigma_{o}=0.4$, characteristic of an elliptical galaxy. However, the inner $3^{\prime \prime}$ are rotating substantially faster, showing the presence of a rapidly rotating core.

Spectra obtained at $\mathrm{H}_{\alpha}$ measure the rotation of the gas in the polar ring. The rotation curve has a constant gradient in the inner region, followed by a well defined turnover at about $10^{\prime \prime}$. Beyond this radius the rotation curve is flat or slightly rising. Perhaps the most surprising fact is that while the ring appears to be a narrow feature at about $12^{\prime \prime}$ radius, $\mathrm{H}_{\alpha}$ emission is actually observed all the way through the nucleus. This suggests that the ring may represent the locus of most intense star formation within an underlying gaseous disk. 


\section{DISCUSSION}

In most polar-ring galaxies, a comparison between the rotational velocities in the ring and in the S0 disk provides a measurement of the flattening of the gravitational potential. In AM2020-5050, however, the low value of $\mathrm{V} / \sigma_{o}$ shows that the inner component is an elliptical galaxy rather than an S0 disk. Since most of the dynamical support comes from the velocity dispersion rather than rotation, a comparison between $\sigma_{o}$ and $\mathrm{V}_{\text {ring }}$ is more relevant.

For bulge-dominated spiral galaxies, Whitmore and Kirshner (1981) find that $\mathrm{V}_{\text {disk }} / \sigma_{o}=1.54 \pm 0.06$. The value for the polar ring of AM2020-5050 is approximately the same, $\mathrm{V}_{\text {ring }} / \sigma_{o}=1.5 \pm 0.1$. This suggests that the rotational velocity of the ring is about the same as it would be in a hypothetical gaseous disk of the central elliptical component. Therefore, the gravitational potential of AM2020-5050 seems to be nearly spherical, even though the shape of the luminous matter is that of an E4 galaxy.

\section{REFERENCES}

Whitmore, B.C., McElroy, D., and Schweizer, F., 1987. Ap. J., submitted.

Whitmore, B.C., and Kirshner, R.P., 1981. Ap. J., 250, 43.

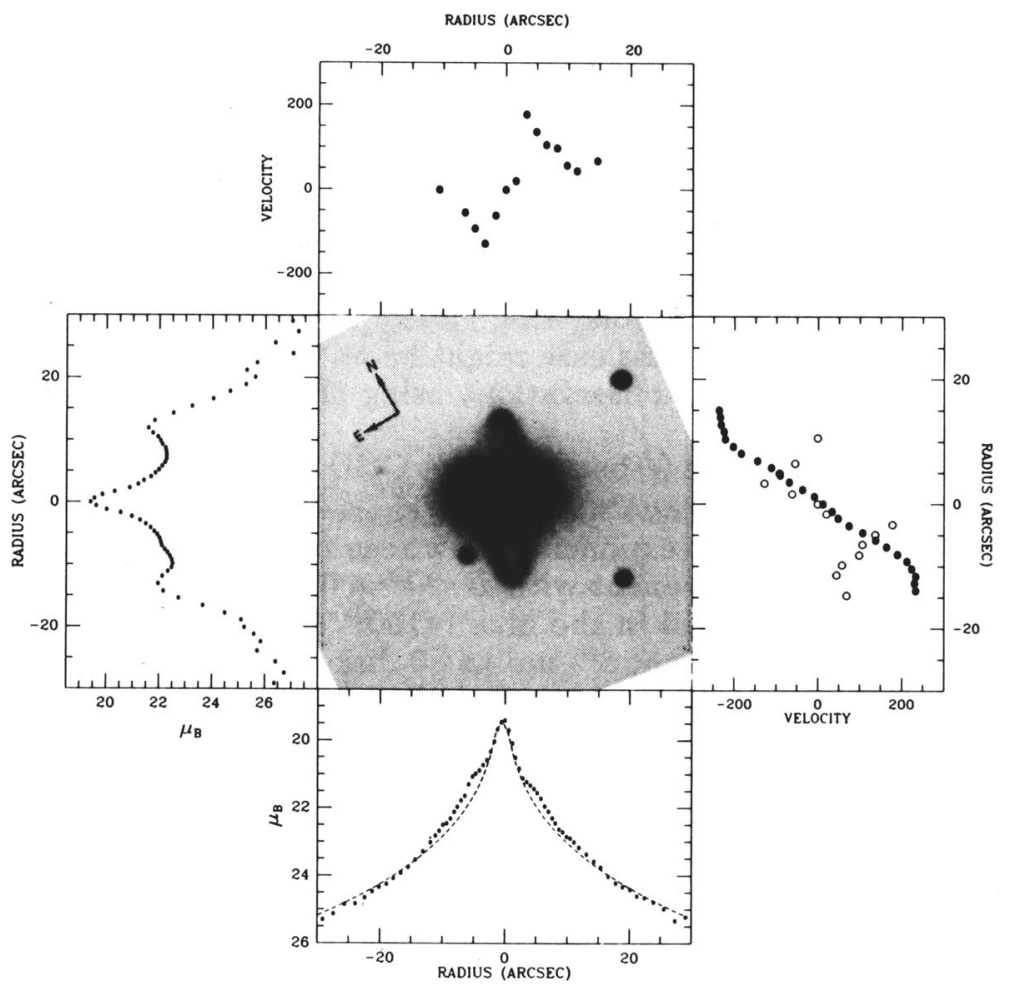

Figure 1. Photometric and Kinematic Observations of AM2020-5050. 